\title{
CODIMENSIONS OF POLYNOMIAL IDENTITIES OF REPRESENTATIONS OF LIE ALGEBRAS
}

\author{
A. S. GORDIENKO \\ (Communicated by Kailash C. Misra)
}

\begin{abstract}
Consider a representation $\rho: L \rightarrow \mathfrak{g l}(V)$ where $L$ is a Lie algebra and $V$ is a finite dimensional vector space. We prove the analog of Amitsur's conjecture on asymptotic behavior for codimensions of polynomial identities of $\rho$.
\end{abstract}

\section{INTRODUCTION}

In the 1980's, conjectures about the asymptotic behaviour of codimensions of polynomial identities were made by S.A. Amitsur and A. Regev. Amitsur's conjecture was proved in 1999 by A. Giambruno and M.V. Zaicev [1, Theorem 6.5.2] for associative algebras and in 2002 by Zaicev [2 for finite dimensional Lie algebras. Alongside polynomial identities of algebras, polynomial identities of representations are also important [3, 4]. Therefore the question arises whether the conjectures hold for codimensions of representations.

Denote by $F\langle X\rangle$ the free associative algebra on the countable set $X=\left\{x_{1}, x_{2}, \ldots\right\}$, i.e. the algebra of polynomials without a constant term in the noncommuting variables $X$ over a field $F$ of characteristic 0 . Let $\rho: L \rightarrow \mathfrak{g l}(V)$ be a linear representation of a Lie algebra $L$ on a vector space $V$ over $F$. Let $f=f\left(x_{1}, \ldots, x_{n}\right) \in F\langle X\rangle$. We say that $f$ is a polynomial identity of $\rho$ if $f\left(\rho\left(a_{1}\right), \ldots, \rho\left(a_{n}\right)\right) v=0$ for all $a_{1}, \ldots, a_{n} \in L, v \in V$. The set $\operatorname{Id}(\rho)$ of polynomial identities of $\rho$ is a two-sided ideal of $F\langle X\rangle$.

Example 1. Let $\rho: \mathfrak{s l}_{2}(F) \rightarrow \mathfrak{g l}(V)$ be any representation of the algebra $\mathfrak{s l}_{2}(F)$. Then

$$
S t_{4}=\sum_{\sigma \in S_{4}}(\operatorname{sign} \sigma) x_{\sigma(1)} x_{\sigma(2)} x_{\sigma(3)} x_{\sigma(4)} \in \operatorname{Id}(\rho),
$$

where $S_{4}$ is the symmetric group on $\{1,2,3,4\}$. Indeed, in order to verify a multilinear identity it is sufficient to substitute basis elements. Since $\operatorname{dim} \mathfrak{s l}_{2}(F)=3$ and $S t_{4}$ is alternating in 4 variables, it vanishes under all evaluations in $\rho\left(\mathfrak{s l}_{2}(F)\right)$.

Received by the editors June 17, 2011 and, in revised form, December 15, 2011.

2010 Mathematics Subject Classification. Primary 17B01; Secondary 16R10, 17B10, 20 C30.

Key words and phrases. Lie algebra, polynomial identity, codimension, cocharacter, symmetric group, Young diagram.

This work was supported by postdoctoral fellowships from the Atlantic Association for Research in Mathematical Sciences (AARMS), the Atlantic Algebra Centre (AAC), Memorial University of Newfoundland (MUN), and the Natural Sciences and Engineering Research Council of Canada (NSERC). 
Let $P_{n}$ be the space of multilinear polynomials in the noncommuting variables $x_{1}, x_{2}, \ldots, x_{n}$. The nonnegative integer $c_{n}(\rho)=\operatorname{dim} \frac{P_{n}}{P_{n} \cap \operatorname{Id}(\rho)}$ is called the $n$th codimension of the representation $\rho$.

Another approach to polynomial identities of representations is concerned with representations of Lie algebras in associative algebras. Let $\tau: L \rightarrow A_{0}$ be a Lie homomorphism where $L$ is a Lie $F$-algebra and $A_{0}$ is an associative $F$-algebra. Let $f=f\left(x_{1}, \ldots, x_{n}\right) \in F\langle X\rangle$. We say that $f$ is a polynomial identity of $\tau$ if $f\left(\tau\left(a_{1}\right), \ldots, \tau\left(a_{n}\right)\right)=0$ for all $a_{1}, \ldots, a_{n} \in L$. Again, the set $\operatorname{Id}(\tau)$ of polynomial identities of $\tau$ is a two-sided ideal of $F\langle X\rangle$. The nonnegative integer $c_{n}(\tau)=$ $\operatorname{dim} \frac{P_{n}}{P_{n} \cap \mathrm{Id}(\tau)}$ is called the $n$th codimension of the representation $\tau$.

These approaches are equivalent. The first one is a partial case of the second one with $A_{0}=\operatorname{End}_{F}(V)$ and $\tau=\rho$. The second one is a partial case of the first one since $\operatorname{Id}(\tau)=\operatorname{Id}(\rho)$, where $\rho: L \rightarrow \mathfrak{g l}_{F}\left(A_{0}+F \cdot 1\right), \rho(a) \cdot b=\tau(a) b, a \in L$, $b \in A_{0}+F \cdot 1$.

Throughout the paper we use the first approach.

Yu. A. Bahturin has suggested that we consider the analog of Amitsur's conjecture for representations.

Conjecture. There exists $\lim _{n \rightarrow \infty} \sqrt[n]{c_{n}(\rho)} \in \mathbb{Z}_{+}$.

Theorem 1. Let $\rho: L \rightarrow \mathfrak{g l}(V)$ be a representation of a Lie algebra $L$ on a finite dimensional vector space $V$ over a field $F$ of characteristic 0 . Then either there exist constants $C_{1}, C_{2}>0, r_{1}, r_{2} \in \mathbb{R}, d \in \mathbb{N}$ such that $C_{1} n^{r_{1}} d^{n} \leqslant c_{n}(\rho) \leqslant C_{2} n^{r_{2}} d^{n}$ for all $n \in \mathbb{N}$ or the equality $c_{n}(\rho)=0$ holds for all sufficiently large $n$.

Corollary. The analog of Amitsur's conjecture holds for such representations.

Our proof follows the outline of the proof by Zaicev 2]. However, in many cases we need to apply new ideas.

Codimensions of representations do not change upon an extension of the base field. The proof is analogous to the cases of codimensions of associative 1, Theorem 4.1.9] and Lie algebras [2, Section 2]. Thus without loss of generality we may assume $F$ to be algebraically closed.

Fix a Levi decomposition $\rho(L)=G+R$, where $G$ is a maximal semisimple subalgebra of $\rho(L)$ and $R$ is a solvable radical of $\rho(L)$. Denote by $A$ the associative subalgebra of $\operatorname{End}_{F}(V)$ generated by $\rho(L)$. Then $A$ becomes a $G$-module under the natural left action by multiplication.

Consider left associative ideals $I_{1}, I_{2}, \ldots, I_{r}, J_{1}, J_{2}, \ldots, J_{r}, r \in \mathbb{Z}_{+}$, of the algebra $A$ such that $J_{k} \subseteq I_{k}$, satisfying the conditions

(1) $I_{k} / J_{k}$ is an irreducible $A$-module or $\operatorname{dim}\left(I_{k} / J_{k}\right)=1$;

(2) for any $G$-submodules $T_{k}$ such that $I_{k}=J_{k} \oplus T_{k}$, there exist elements $q_{1}$, $\ldots, q_{r-1} \in A \cup\{1\}$ such that $T_{1} q_{1} T_{2} \ldots T_{r-1} q_{r-1} T_{r} \neq 0$.

Let $M$ be a left $A$-module. Denote by Ann $M$ its annihilator in $A$. Let

$$
d=d(\rho)=\max \left(\operatorname{dim} \frac{\rho(L)}{\rho(L) \cap \operatorname{Ann}\left(I_{1} / J_{1}\right) \cap \cdots \cap \operatorname{Ann}\left(I_{r} / J_{r}\right)}\right),
$$

where the maximum is found among all $r \in \mathbb{Z}_{+}$and all $I_{1}, \ldots, I_{r}, J_{1}, \ldots, J_{r}$ satisfying Conditions (1)-(2). We claim that $d(\rho)$ coincides with $d$ from Theorem 1 .

We need several auxiliary lemmas.

Lemma 1. $[\rho(L), R] \subseteq J(A)$, where $J(A)$ is the Jacobson radical of $A$. 
Proof. Let $V=V_{0} \supseteq V_{1} \supseteq V_{2} \supseteq \ldots \supseteq V_{t}=\{0\}$ be a composition chain in $V$. Then each $V_{i} / V_{i+1}$ is an irreducible $\rho(L)$-module. Denote the corresponding homomorphism by $\varphi_{i}: \rho(L) \rightarrow \mathfrak{g l}\left(V_{i} / V_{i+1}\right)$. Then by E. Cartan's theorem [5, Proposition 1.4.11], $\varphi_{i}(\rho(L))$ is semisimple or the direct sum of a semisimple ideal and the center of $\mathfrak{g l}\left(V_{i} / V_{i+1}\right)$. Thus $\varphi_{i}([\rho(L), \rho(L)])$ is semisimple and $\varphi_{i}([\rho(L), \rho(L)] \cap R)=$ 0 . Since $[\rho(L), R] \subseteq[\rho(L), \rho(L)] \cap R$, we have $\varphi_{i}([\rho(L), R])=0$ and $[\rho(L), R] V_{i} \subseteq$ $V_{i+1}$. Therefore the associative ideal of $A$ generated by $[\rho(L), R]$ is nilpotent since $a_{1}\left(b_{11} \ldots b_{1 k_{1}}\right) a_{2}\left(b_{21} \ldots b_{2 k_{2}}\right) a_{3} \ldots a_{t}$ is a zero operator on $V$ for all $a_{i} \in[\rho(L), R]$ and $b_{i j} \in \rho(L)$. Thus $[\rho(L), R] \subseteq J(A)$.

Lemma 2. There exists a subspace $S \subseteq R$ such that $R=S+\rho(L) \cap J(A)$ is the direct sum of subspaces and $[G, S]=0$.

Proof. There is a natural ad-action of $G$ on $R:(\operatorname{ad} g) a=[g, a], g \in G, a \in R$. Since $\rho(L) \cap J(A)$ is a submodule of $R$ and $G$ is semisimple, there exists a complementary $G$-submodule $S$. Thus $[G, S] \subseteq S$. By virtue of Lemma $1,[G, S] \subseteq[\rho(L), R] \subseteq$ $\rho(L) \cap J(A)$. Therefore, $[G, S]=0$.

Lemma 3. Let $M$ be an irreducible A-module. Then either $M$ is an irreducible $G$-module or $\operatorname{dim} M=1$. Furthermore, $S$ acts on $M$ by scalar operators.

Proof. Note that $M$ is an irreducible $\rho(L)$-module. By Lemma 1, $[\rho(L), R] \subseteq$ $\rho(L) \cap J(A)$. Therefore $[\rho(L), R] M \subseteq J(A) M=0$ and, by virtue of Schur's lemma, $S \subseteq R$ acts by scalar operators on $M$. Thus all subspaces in $M$ invariant under the action of $G$ are invariant under the action of $\rho(L)$.

Lemma 4. Let $M$ be an irreducible A-module. Then

$$
\operatorname{Ann}_{\rho(L)} M=\operatorname{Ann}_{G} M+\operatorname{Ann}_{S} M+(\rho(L) \cap J(A)) .
$$

Proof. If $M$ is an irreducible $A$-module, then $J(A) M=0$. Thus it is sufficient to prove that if $g+s \in \operatorname{Ann}_{\rho(L)} M, g \in G, s \in S$, then $g \in \operatorname{Ann}_{G} M$ and $s \in \operatorname{Ann}_{S} M$. Denote $\varphi: \rho(L) \rightarrow \mathfrak{g l}(M)$. Note that $s m=-g m$ for all $m \in M$ since $g+s \in$ $\operatorname{Ann}_{\rho(L)} M$. By Lemma 3, $s$ is a scalar operator on $M$. Therefore, $g$ is a scalar operator on $M$ too, and $\varphi(g)$ belongs to the center of the semisimple algebra $\varphi(G)$. Hence $\varphi(g)=0$, and this finishes the proof of the lemma.

One of the main tools in the investigation of polynomial identities is provided by the representation theory of symmetric groups. The symmetric group $S_{n}$ acts on the space $\frac{P_{n}}{P_{n} \cap \operatorname{Id}(\rho)}$ by permuting the variables. Irreducible $F S_{n}$-modules are described by partitions $\lambda=\left(\lambda_{1}, \ldots, \lambda_{s}\right) \vdash n$ and their Young diagrams $D_{\lambda}$. The character $\chi_{n}(\rho)$ of the $F S_{n}$-module $\frac{P_{n}}{P_{n} \cap \operatorname{Id}(\rho)}$ is called the $n$th cocharacter of the representation $\rho$. We can rewrite it as a sum $\chi_{n}(\rho)=\sum_{\lambda \vdash n} m(\rho, \lambda) \chi(\lambda)$ of irreducible characters $\chi(\lambda)$. Let $e_{T_{\lambda}}=a_{T_{\lambda}} b_{T_{\lambda}}$ and $e_{T_{\lambda}}^{*}=b_{T_{\lambda}} a_{T_{\lambda}}$ (where $a_{T_{\lambda}}=\sum_{\pi \in R_{T_{\lambda}}} \pi$ and $b_{T_{\lambda}}=$ $\left.\sum_{\sigma \in C_{T_{\lambda}}}(\operatorname{sign} \sigma) \sigma\right)$ be the Young symmetrizers corresponding to a Young tableau $T_{\lambda}$. Then $M(\lambda)=F S e_{T_{\lambda}} \cong F S e_{T_{\lambda}}^{*}$ is an irreducible $F S_{n}$-module corresponding to a partition $\lambda \vdash n$. We refer the reader to [1, 6, 7] for accounts of $S_{n}$-representations and their applications to polynomial identities. 


\section{UPPER BOUND}

Fix a composition chain of $A$-submodules

$$
A=B_{0} \supsetneqq B_{1} \supsetneqq B_{2} \supsetneqq \cdots \supsetneqq J(A) \supsetneqq \cdots \supsetneqq B_{\theta-1} \supsetneqq B_{\theta}=\{0\}
$$

in the module $A$. Let ht $a=\max _{a \in B_{k}} k$ for $a \in A$.

Remark. If $d=d(\rho)=0$, then $\rho(L) \subseteq \operatorname{Ann}\left(B_{i-1} / B_{i}\right)$ for all $1 \leqslant i \leqslant \theta$ and $a_{1} a_{2} \ldots a_{n}=0$ for all $a_{i} \in \rho(L)$ and $n \geqslant \theta+1$. Thus $c_{n}(\rho)=0$ for all $n \geqslant \theta+1$. Therefore we assume $d>0$.

Let $Y=\left\{y_{11}, y_{12}, \ldots, y_{1 j_{1}} ; y_{21}, y_{22}, \ldots, y_{2 j_{2}} ; \ldots ; y_{m 1}, y_{m 2}, \ldots, y_{m j_{m}}\right\}, Y_{1}, \ldots$, $Y_{q}$, and $\left\{z_{1}, \ldots, z_{m}\right\}$ be subsets of $\left\{x_{1}, x_{2}, \ldots, x_{n}\right\}$ such that $Y_{i} \subseteq Y,\left|Y_{i}\right|=d+1$, $Y_{i} \cap Y_{j}=\varnothing$ for $i \neq j, Y \cap\left\{z_{1}, \ldots, z_{m}\right\}=\varnothing, j_{i} \geqslant 0$. Denote

$$
g_{m, q}=\operatorname{Alt}_{1} \ldots \operatorname{Alt}_{q}\left(\left(y_{11} y_{12} \ldots y_{1 j_{1}}\right) z_{1}\left(y_{21} y_{22} \ldots y_{2 j_{2}}\right) z_{2} \ldots\left(y_{m 1} y_{m 2} \ldots y_{m j_{m}}\right) z_{m}\right) \text {, }
$$

where $\mathrm{Alt}_{i}$ is the operator of alternation on the variables of $Y_{i}$.

Let $\varphi: F\langle X\rangle \rightarrow A$ be a homomorphism induced by a substitution $\left\{x_{1}, x_{2}, \ldots, x_{n}\right\}$ $\rightarrow \rho(L)$. We say that $\varphi$ is proper for $g_{m, q}$ if $\varphi\left(z_{i}\right) \in \rho(L) \cap J(A)$ for $1 \leqslant i \leqslant m-1$, $\varphi\left(z_{m}\right) \in(\rho(L) \cap J(A)) \cup G \cup S$, and $\varphi\left(y_{i k}\right) \in G \cup S$ for $1 \leqslant i \leqslant m, 1 \leqslant k \leqslant j_{i}$.

Lemma 5. Let $\varphi$ be a proper homomorphism for $g_{m, q}$. Then $\varphi\left(g_{m, q}\right)$ can be rewritten as a sum of $\psi\left(g_{m+1, q^{\prime}}\right)$, where $\psi$ is a proper homomorphism for $g_{m+1, q^{\prime}}$, $q^{\prime} \geqslant q-(\operatorname{dim} A) m-2$. ( $Y^{\prime}, Y_{i}^{\prime}, z_{1}^{\prime}, \ldots, z_{m+1}^{\prime}$ may be different for different terms.)

Proof. Let $\alpha_{i}=$ ht $\varphi\left(z_{i}\right)$. We will use induction on $\sum_{i=1}^{m} \alpha_{i}$. (The sum will grow.) Note that $\alpha_{i} \leqslant \theta \leqslant \operatorname{dim} A$. Denote $I_{i}=B_{\alpha_{i}}, J_{i}=B_{\alpha_{i}}+1$.

First, consider the case when $I_{1}, \ldots, I_{m}, J_{1}, \ldots, J_{m}$ do not satisfy Conditions (1)-(2). In this case we can choose $G$-submodules $T_{i}, I_{i}=T_{i} \oplus J_{i}$ such that

$$
T_{1} h_{1} T_{2} h_{2} \ldots h_{m-1} T_{m}=0
$$

for all $h_{i} \in A \cup\{1\}$. Rewrite $\varphi\left(z_{i}\right)=a_{i}^{\prime}+a_{i}^{\prime \prime}, a_{i}^{\prime} \in T_{i}, a_{i}^{\prime \prime} \in J_{i}$. Note that ht $a_{i}^{\prime \prime}>$ ht $\varphi\left(z_{i}\right)$. Since $g_{m, q}$ is multilinear, we can rewrite $\varphi\left(g_{m, q}\right)$ as a sum of similar terms $\tilde{\varphi}\left(g_{m, q}\right)$, where $\tilde{\varphi}\left(z_{i}\right)$ equals either $a_{i}^{\prime}$ or $a_{i}^{\prime \prime}$. The term where all $\tilde{\varphi}\left(z_{i}\right)=a_{i}^{\prime} \in T_{i}$ equals 0 since $I_{1}, \ldots, I_{m}, J_{1}, \ldots, J_{m}$ do not satisfy Conditions (1)-(2). For the other terms $\tilde{\varphi}\left(g_{m, q}\right)$ we have $\sum_{i=1}^{m}$ ht $\tilde{\varphi}\left(z_{i}\right)>\sum_{i=1}^{m}$ ht $\varphi\left(z_{i}\right)$.

Thus without loss of generality we may assume that $I_{1}, \ldots, I_{m}, J_{1}, \ldots, J_{m}$ satisfy Conditions (1)-(2). In this case, $\operatorname{dim}\left(\rho(L) \cap \operatorname{Ann}\left(I_{1} / J_{1}\right) \cap \ldots \cap \operatorname{Ann}\left(I_{m} / J_{m}\right)\right) \geqslant$ $\operatorname{dim}(\rho(L))-d$. By virtue of Lemma 4 .

$$
\begin{aligned}
\rho(L) & \cap \operatorname{Ann}\left(I_{1} / J_{1}\right) \cap \ldots \cap \operatorname{Ann}\left(I_{m} / J_{m}\right) \\
= & G \cap \operatorname{Ann}\left(I_{1} / J_{1}\right) \cap \ldots \cap \operatorname{Ann}\left(I_{m} / J_{m}\right) \\
& +S \cap \operatorname{Ann}\left(I_{1} / J_{1}\right) \cap \ldots \cap \operatorname{Ann}\left(I_{m} / J_{m}\right)+\rho(L) \cap J(A) .
\end{aligned}
$$

Choose a basis in $G$ that includes a basis of $G \cap \operatorname{Ann}\left(I_{1} / J_{1}\right) \cap \ldots \cap \operatorname{Ann}\left(I_{m} / J_{m}\right)$ and a basis in $S$ that includes a basis of $S \cap \operatorname{Ann}\left(I_{1} / J_{1}\right) \cap \ldots \cap \operatorname{Ann}\left(I_{m} / J_{m}\right)$. Since $g_{m, q}$ is multilinear, we may assume that only basis elements are substituted for $y_{k \ell}$. Note that $g_{m, q}$ is alternating in $Y_{i}$. Hence, if $\varphi\left(g_{m, q}\right) \neq 0$, then for every $1 \leqslant i \leqslant q$ there exists $y_{k \ell} \in Y_{i}$ such that either $\varphi\left(y_{k \ell}\right) \in G \cap \operatorname{Ann}\left(I_{1} / J_{1}\right) \cap \ldots \cap \operatorname{Ann}\left(I_{m} / J_{m}\right)$ or $\varphi\left(y_{k \ell}\right) \in S \cap \operatorname{Ann}\left(I_{1} / J_{1}\right) \cap \ldots \cap \operatorname{Ann}\left(I_{m} / J_{m}\right)$. 
Consider the case when $\varphi\left(y_{k \ell}\right) \in G \cap \operatorname{Ann}\left(I_{1} / J_{1}\right) \cap \ldots \cap \operatorname{Ann}\left(I_{m} / J_{m}\right)$ for some $y_{k \ell}$. We can choose $G$-submodules $T_{k}$ such that $I_{k}=T_{k} \oplus J_{k}$. We may assume that $\varphi\left(z_{k}\right) \in T_{k}$ since elements of $J_{k}$ have greater heights. Therefore $a \varphi\left(z_{k}\right) \in T_{k} \cap J_{k}$ for all $a \in G \cap \operatorname{Ann}\left(I_{1} / J_{1}\right) \cap \ldots \cap \operatorname{Ann}\left(I_{m} / J_{m}\right)$. Hence $a \varphi\left(z_{k}\right)=0$. Moreover, $G \cap \operatorname{Ann}\left(I_{1} / J_{1}\right) \cap \ldots \cap \operatorname{Ann}\left(I_{m} / J_{m}\right)$ is a Lie ideal of $G$ and $[G, S]=0$. Thus $\varphi\left(y_{k 1} \ldots y_{k j_{k}} z_{k}\right)=0$. Expanding the alternations, we obtain $\varphi\left(g_{m, q}\right)=0$.

Consider the case when $\varphi\left(y_{k \ell}\right) \in S \cap \operatorname{Ann}\left(I_{1} / J_{1}\right) \cap \ldots \cap \operatorname{Ann}\left(I_{m} / J_{m}\right)$ for some $y_{k \ell} \in Y_{q}$. Expand the alternation Alt $_{q}$ in $g_{m, q}$ and rewrite $g_{m, q}$ as a sum of

$$
\begin{array}{r}
\tilde{g}_{m, q-1}=\operatorname{Alt}_{1} \ldots \operatorname{Alt}_{q-1}\left(\left(y_{11} y_{12} \ldots y_{1 j_{1}}\right) z_{1}\left(y_{21} y_{22} \ldots y_{2 j_{2}}\right) z_{2}\right. \\
\left.\ldots\left(y_{m 1} y_{m 2} \ldots y_{m j_{m}}\right) z_{m}\right) .
\end{array}
$$

The operator $\mathrm{Alt}_{q}$ may change indices; however we keep the notation $y_{k \ell}$ for the variable with the property $\varphi\left(y_{k \ell}\right) \in S \cap \operatorname{Ann}\left(I_{1} / J_{1}\right) \cap \ldots \cap \operatorname{Ann}\left(I_{m} / J_{m}\right)$. Now the alternation does not affect $y_{k \ell}$. Note that

$$
\begin{gathered}
y_{k 1} y_{k 2} \ldots y_{k j_{k}} z_{k}=y_{k 1} y_{k 2} \ldots y_{k, \ell-1} y_{k, \ell+1} \ldots y_{k j_{k}} y_{k \ell} z_{k} \\
+\sum_{\beta=\ell+1}^{j_{k}} y_{k 1} y_{k 2} \ldots y_{k, \ell-1} y_{k, \ell+1} \ldots y_{k, \beta-1}\left[y_{k \ell}, y_{k \beta}\right] y_{k, \beta+1} \ldots y_{k j_{k}} z_{k} .
\end{gathered}
$$

In the first polynomial on the right hand side we replace $y_{k \ell} z_{k}$ with $z_{k}^{\prime}$ and define $\varphi^{\prime}\left(z_{k}^{\prime}\right)=\varphi\left(y_{k \ell}\right) \varphi\left(z_{k}\right), \varphi^{\prime}(x)=\varphi(x)$ for other variables $x$. Then ht $\varphi^{\prime}\left(z_{k}^{\prime}\right)>\operatorname{ht} \varphi\left(z_{k}\right)$ and we can use the inductive assumption. If $y_{k \beta} \in Y_{j}$ for some $j$, then we expand the alternation $\mathrm{Alt}_{j}$ in this term in $\tilde{g}_{m, q-1}$. If $\varphi\left(y_{k \beta}\right) \in G$, then this term is zero. If $\varphi\left(y_{k \beta}\right) \in S$, then $\varphi\left(\left[y_{k \ell}, y_{k \beta}\right]\right) \in \rho(L) \cap J(A)$. We replace $\left[y_{k \ell}, y_{k \beta}\right]$ with an additional variable $z_{m+1}^{\prime}$ and define $\psi\left(z_{m+1}^{\prime}\right)=\varphi\left(\left[y_{k \ell}, y_{k \beta}\right]\right), \psi(x)=\varphi(x)$ for other variables $x$. Then the polynomial has the desired form. In each inductive step we reduce $q$ by no more than 1 , and the maximal number of inductive steps equals $(\operatorname{dim} A) m$. This finishes the proof.

Since the Jacobson radical is a nilpotent ideal, $J(A)^{p}=0$ for some $p \in \mathbb{N}$.

Lemma 6. If $\lambda=\left(\lambda_{1}, \ldots, \lambda_{s}\right) \vdash n$ and $\lambda_{d+1} \geqslant p((\operatorname{dim} A) p+3)$ or $\lambda_{\operatorname{dim} \rho(L)+1}>0$, then $m(\rho, \lambda)=0$.

Proof. It is sufficient to prove that $e_{T_{\lambda}}^{*} f \in \operatorname{Id}(\rho)$ for every $f \in P_{n}$ and a Young tableau $T_{\lambda}, \lambda \vdash n$, with $\lambda_{d+1} \geqslant p((\operatorname{dim} A) p+3)$ or $\lambda_{\operatorname{dim} \rho(L)+1}>0$.

Fix some basis of $\rho(L)$ that is a union of bases of $G, S$, and $\rho(L) \cap J(A)$. Since polynomials are multilinear, it is sufficient to substitute only basis elements. Note that $e_{T_{\lambda}}^{*}=b_{T_{\lambda}} a_{T_{\lambda}}$ and $b_{T_{\lambda}}$ alternates the variables of each column of $T_{\lambda}$. Hence if we make a substitution and $e_{T_{\lambda}}^{*} f$ does not vanish, then this implies that different basis elements are substituted for the variables of each column. But if $\lambda_{\operatorname{dim} \rho(L)+1}>0$, then the length of the first column is greater than $\operatorname{dim} \rho(L)$. Therefore, $e_{T_{\lambda}}^{*} f \in$ $\operatorname{Id}(\rho)$.

Consider the case $\lambda_{d+1} \geqslant p((\operatorname{dim} A) p+3)$. Let $\varphi$ be a substitution of basis elements for the variables $x_{1}, \ldots, x_{n}$. Then $e_{T_{\lambda}}^{*} f$ can be rewritten as a sum of polynomials $g_{m, q}$ where $1 \leqslant m \leqslant p, q \geqslant p((\operatorname{dim} A) p+2)$, and $z_{i}, 1 \leqslant i \leqslant m-1$, are replaced with elements of $\rho(L) \cap J(A)$. (For different terms $g_{m, q}$, the numbers $m$ and $q$, variables $z_{i}, y_{i j}$, and sets $Y_{i}$ can be different.) Indeed, we expand symmetrization 
on all variables and alternation on the variables replaced with $\rho(L) \cap J(A)$. Also, we expand the alternation on the set that includes the last variable, which we denote by $z_{m}$.

Applying Lemma 5 many times, we increase $m$. The ideal $J(A)$ is nilpotent and $\varphi\left(g_{p+1, q}\right)=0$ for every $q$ and proper homomorphism $\varphi$. Reducing $q$ by no more than $p((\operatorname{dim} A) p+2)$, we obtain $\varphi\left(e_{T_{\lambda}}^{*} f\right)=0$.

Now we can prove

Theorem 2. If $d>0$, then there exist constants $C_{2}>0, r_{2} \in \mathbb{R}$ such that $c_{n}(\rho) \leqslant C_{2} n^{r_{2}} d^{n}$ for all $n \in \mathbb{N}$. In the case $d=0$, the equality $c_{n}(\rho)=0$ holds for all sufficiently large $n$.

Proof. Lemma 6] and [1, Lemmas 6.2.4, 6.2.5] imply

$$
\sum_{m(\rho, \lambda) \neq 0} \operatorname{dim} M(\lambda) \leqslant C_{3} n^{r_{3}} d^{n}
$$

for some constants $C_{3}, r_{3}>0$. Denote by $\operatorname{Id}(A) \subseteq F\langle X\rangle$ the ideal of polynomial identities of the associative algebra $A$. Let $\chi_{n}(A)=\chi\left(\frac{P_{n}}{P_{n} \cap \operatorname{Id}(A)}\right)=$ $\sum_{\lambda} m(A, \lambda) \chi(\lambda)$. Then $\operatorname{Id}(A) \subseteq \operatorname{Id}(\rho)$ and $m(\rho, \lambda) \leqslant m(A, \lambda) \leqslant C_{4} n^{r_{4}}$ for some $C_{4}, r_{4}>0$ by [1, Lemma 4.9.2]. Together with (10) this implies the upper bound.

\section{LOWER BOUND}

By the definition of $d$, there exist left associative ideals $I_{1}, I_{2}, \ldots, I_{r}, J_{1}, J_{2}$, $\ldots, J_{r}, r \in \mathbb{Z}_{+}$, of the algebra $A$, satisfying Conditions (1)-(2), $J_{k} \subseteq I_{k}$, such that

$$
d=\operatorname{dim} \frac{\rho(L)}{\rho(L) \cap \operatorname{Ann}\left(I_{1} / J_{1}\right) \cap \cdots \cap \operatorname{Ann}\left(I_{r} / J_{r}\right)} .
$$

We consider the case $d>0$.

Without loss of generality we may assume that

$$
\rho(L) \cap \bigcap_{k=1}^{r} \operatorname{Ann}\left(I_{k} / J_{k}\right) \neq \rho(L) \cap \bigcap_{\substack{k=1, k \neq \ell}}^{r} \operatorname{Ann}\left(I_{k} / J_{k}\right)
$$

for all $1 \leqslant \ell \leqslant r$. In particular, $\rho(L)$ has nonzero action on each $I_{k} / J_{k}$.

Our aim is to present a partition $\lambda \vdash n$ with $m(\rho, \lambda) \neq 0$ such that $\operatorname{dim} M(\lambda)$ has the desired asymptotic behavior. We will glue alternating polynomials constructed by Yu.P. Razmyslov's theorem from faithful irreducible modules over reductive algebras. In order to do this, we have to choose the reductive algebras.

Lemma 7. There exist Lie ideals $G_{1}, \ldots, G_{r}$ in $G$ and elements $a_{1}, \ldots, a_{r} \in S$ (some of $a_{i}$ and $G_{j}$ may be zero) such that

(1) $G_{1}+\ldots+G_{r}=G_{1} \oplus \ldots \oplus G_{r}$;

(2) $\left\langle a_{1}, a_{2}, \ldots, a_{r}\right\rangle_{F}=\left\langle a_{1}\right\rangle_{F} \oplus \ldots \oplus\left\langle a_{r}\right\rangle_{F}$;

(3) $\sum_{k=1}^{r}\left(\operatorname{dim}\left(G_{k} \oplus\left\langle a_{k}\right\rangle_{F}\right)=d\right.$;

(4) $I_{k} / J_{k}$ is a faithful $G_{k} \oplus\left\langle a_{k}\right\rangle_{F}$-module;

(5) $G_{i} I_{k} / J_{k}=a_{i} I_{k} / J_{k}=0$ for $i>k$. 
Proof. Consider $N_{\ell}=\rho(L) \cap \bigcap_{k=1}^{\ell} \operatorname{Ann}\left(I_{k} / J_{k}\right), 1 \leqslant \ell \leqslant r, N_{0}=\rho(L)$. Since $G$ is semisimple, we can choose such ideals $G_{\ell}$ that $N_{\ell-1} \cap G=G_{\ell} \oplus\left(N_{\ell} \cap G\right)$. Note that $\operatorname{dim}\left(S / \operatorname{Ann}_{S}\left(I_{\ell} / J_{\ell}\right)\right) \leqslant 1$ since $S$ acts by scalar operators by Lemma 3. Thus we can choose elements $a_{\ell}$ such that $N_{\ell-1} \cap S=\left\langle a_{\ell}\right\rangle_{F} \oplus\left(N_{\ell} \cap G\right)$. Hence Properties (1), (2), (4), (5) hold. By Lemma 4, $N_{k}=N_{k} \cap G+N_{k} \cap S+\rho(L) \cap J(A)$. Therefore,

$$
N_{\ell-1}=G_{\ell}+N_{\ell} \cap G+\left\langle a_{\ell}\right\rangle_{F}+N_{\ell} \cap S+\rho(L) \cap J(A)=\left(G_{\ell}+\left\langle a_{\ell}\right\rangle_{F}\right)+N_{\ell}
$$

(a direct sum of subspaces) and Property (3) holds.

Lemma 8. For every $1 \leqslant i \leqslant r$ there exists a decomposition $a_{i}=b_{i}+c_{i}$ such that $c_{i} \in A$ acts as a diagonalizable operator on $A$ and $b_{i} \in J(A)$. Furthermore, elements $c_{i}$ commute with each other and $b_{i}$ and $c_{i}$ are polynomials in $a_{1}, \ldots, a_{r}$.

Proof. The solvable Lie algebra $R+J(A)$ acts on the algebra $\tilde{A}=A+F \cdot 1$ by left multiplication. By virtue of the Lie theorem, there exists a basis in $\tilde{A}$ in which all the operators from $R+J(A)$ have upper triangular matrices. Denote the corresponding embedding $A \hookrightarrow M_{m}(F)$ by $\psi$. Here $m=\operatorname{dim} \tilde{A}$.

Let $A_{1}$ be the associative algebra generated by $a_{1}, \ldots, a_{r}$. Since $\psi(R) \subseteq \mathfrak{t}_{m}(F)$, we have $\psi\left(A_{1}\right) \subseteq U T_{m}(F)$. Here $U T_{m}(F)$ is the associative algebra of upper triangular matrices $m \times m$. There is a decomposition $U T_{m}(F)=F e_{11}+F e_{22}+$ $\cdots+F e_{m m}+N$ where $N=\left\langle e_{i j} \mid 1 \leqslant i<j \leqslant m\right\rangle_{F}$ is a nilpotent ideal. Thus there is no subalgebra in $A_{1}$ isomorphic to $M_{2}(F)$ and, by the Wedderburn-Malcev theorem, $A_{1}=F e_{1}+\cdots+F e_{t}+J\left(A_{1}\right)$ (the direct sum of subspaces) for some idempotents $e_{i} \in A_{1}$. Denote for every $a_{i}$ its component in $J\left(A_{1}\right)$ by $b_{i}$ and its component in $F e_{1} \oplus \cdots \oplus F e_{t}$ by $c_{i}$. Note that $e_{i}$ are commuting diagonalizable operators. Thus they have a common basis of eigenvectors in $\tilde{A}$, and $c_{i}$ are commuting diagonalizable operators, too.

We claim that the space $J\left(A_{1}\right)+J(A)$ generates a nilpotent ideal $I$ in $A$. First, $\psi\left(J\left(A_{1}\right)\right), \psi(J(A)) \subseteq U T_{m}(F)$ and consist of nilpotent elements. Thus, the corresponding matrices have zero diagonal elements and $\psi\left(J\left(A_{1}\right)\right), \psi(J(A)) \subseteq N$. Denote $N_{k}=\left\langle e_{i j} \mid i+k \leqslant j\right\rangle_{F} \subseteq N$. Then

$$
N=N_{1} \supsetneqq N_{2} \supsetneqq \cdots \supsetneqq N_{m-1} \supsetneqq N_{m}=\{0\} .
$$

Let $\operatorname{ht}_{N} a=k$ if $\psi(a) \in N_{k}, \psi(a) \notin N_{k+1}$.

Recall that $(J(A))^{p}=0$. We claim that $I^{m+p}=0$. The space $I^{m+p}$ is a span of $h_{1} j_{1} h_{2} j_{2} \ldots j_{m+p} h_{m+p+1}$ where $j_{k} \in J\left(A_{1}\right) \cup J(A)$ and $h_{k} \in A \cup\{1\}$. If at least $p$ elements $j_{k}$ belong to $J(A)$, then the product equals 0 . Thus we may assume that at least $m$ elements $j_{k}$ belong to $J_{1}(A)$.

Let $j_{i} \in J\left(A_{1}\right), h_{i} \in A \cup\{1\}$. We prove by induction on $\ell$ that $j_{1} h_{1} j_{2} h_{2} \ldots h_{\ell-1} j_{\ell}$ can be expressed as a sum of $\tilde{j}_{1} \tilde{j}_{2} \ldots \tilde{j}_{\alpha} j_{1}^{\prime} j_{2}^{\prime} \ldots j_{\beta}^{\prime} a$ where $\tilde{j}_{i} \in J\left(A_{1}\right), j_{i}^{\prime} \in J(A)$, $a \in A \cup\{1\}$, and $\alpha+\sum_{i=1}^{\beta} \mathrm{ht}_{N} j_{i}^{\prime} \geqslant \ell$. Indeed, suppose that $j_{1} h_{1} j_{2} h_{2} \ldots h_{\ell-2} j_{\ell-1}$ can be expressed as a sum of $\tilde{j}_{1} \tilde{j}_{2} \ldots \tilde{j}_{\gamma} j_{1}^{\prime} j_{2}^{\prime} \ldots j_{\varkappa}^{\prime} a$ where $\tilde{j}_{i} \in J\left(A_{1}\right), j_{i}^{\prime} \in J(A)$, $a \in \tilde{A}$, and $\gamma+\sum_{i=1}^{\varkappa} \mathrm{ht}_{N} j_{i}^{\prime} \geqslant \ell-1$. Then $j_{1} h_{1} j_{2} h_{2} \ldots j_{\ell-1} h_{\ell-1} j_{\ell}$ is a sum of

$$
\begin{aligned}
\tilde{j}_{1} \tilde{j}_{2} \ldots \tilde{j}_{\gamma} j_{1}^{\prime} j_{2}^{\prime} \ldots j_{\varkappa}^{\prime} a h_{\ell-1} j_{\ell}= & \tilde{j}_{1} \tilde{j}_{2} \ldots \tilde{j}_{\gamma} j_{1}^{\prime} j_{2}^{\prime} \ldots j_{\varkappa}^{\prime}\left[a h_{\ell-1}, j_{\ell}\right] \\
& +\tilde{j}_{1} \tilde{j}_{2} \ldots \tilde{j}_{\gamma} j_{1}^{\prime} j_{2}^{\prime} \ldots j_{\varkappa}^{\prime} j_{\ell}\left(a h_{\ell-1}\right) .
\end{aligned}
$$


Note that, by virtue of the Jacobi identity and Lemma 1, $\left[a h_{\ell-1}, j_{\ell}\right] \in J(A)$. Thus it is sufficient to consider only the second term in the right hand side. However,

$$
\begin{gathered}
\tilde{j}_{1} \tilde{j}_{2} \ldots \tilde{j}_{\gamma} j_{1}^{\prime} j_{2}^{\prime} \ldots j_{\varkappa}^{\prime} j_{\ell}\left(a h_{\ell-1}\right)=\tilde{j}_{1} \tilde{j}_{2} \ldots \tilde{j}_{\gamma} j_{\ell} j_{1}^{\prime} j_{2}^{\prime} \ldots j_{\varkappa}^{\prime}\left(a h_{\ell-1}\right) \\
+\sum_{i=1}^{\varkappa} \tilde{j}_{1} \tilde{j}_{2} \ldots \tilde{j}_{\gamma} j_{1}^{\prime} j_{2}^{\prime} \ldots j_{i-1}^{\prime}\left[j_{i}^{\prime}, j_{\ell}\right] j_{i+1}^{\prime} \ldots j_{\varkappa}^{\prime}\left(a h_{\ell-1}\right) .
\end{gathered}
$$

Since $\left[j_{i}^{\prime}, j_{\ell}\right] \in J(A)$ and $\mathrm{ht}_{N}\left[j_{i}^{\prime}, j_{\ell}\right] \geqslant 1+\mathrm{ht}_{N} j_{i}^{\prime}$, all the terms have the desired form. Therefore,

$$
j_{1} h_{1} j_{2} h_{2} \ldots j_{m-1} h_{m-1} j_{m} \in \psi^{-1}\left(N_{m}\right)=\{0\},
$$

$I^{m+p}=0$, and

$$
J(A) \subseteq J\left(A_{1}\right)+J(A) \subseteq I \subseteq J(A) .
$$

In particular, $b_{i} \in J\left(A_{1}\right) \subseteq J(A)$.

Lemma 9. There exist complementary subspaces $I_{k}=\tilde{T}_{k} \oplus J_{k}$ such that

(1) $\tilde{T}_{k}$ is an irreducible $G \oplus\left\langle c_{1}, \ldots, c_{r}\right\rangle_{F}$-submodule;

(2) $c_{i}$ acts on each $\tilde{T}_{k}$ as a scalar operator;

(3) $\tilde{T}_{k}$ is a faithful $G_{k} \oplus\left\langle c_{k}\right\rangle_{F}$-module. Moreover, $\sum_{k=1}^{r} \operatorname{dim}\left(G_{k} \oplus\left\langle c_{k}\right\rangle_{F}\right)=d$.

(4) $G_{i} \tilde{T}_{k}=c_{i} \tilde{T}_{k}=0$ for $i>k$.

Proof. The elements $c_{i}$ are diagonalizable on $A$ and commute. Therefore, an eigenspace of any $c_{i}$ is invariant under the action of other $c_{k}$. Using induction, we split $A=\bigoplus_{i=1}^{\alpha} W_{i}$, where $W_{i}$ are intersections of eigenspaces of $c_{k}$ and elements $c_{k}$ act as scalar operators on $W_{i}$. By virtue of Lemma 1 and the Jacobi identity, $\left[c_{i}, G\right]=0$. Thus $W_{i}$ are $G$-submodules and $A$ is a completely reducible $G \oplus\left\langle c_{1}, \ldots, c_{r}\right\rangle_{F}$-module. Therefore, for every $J_{k}$ we can choose a complementary $G \oplus\left\langle c_{1}, \ldots, c_{r}\right\rangle_{F}$-submodule $\tilde{T}_{k}$ in $I_{k}$. Then $\tilde{T}_{k} \cong I_{k} / J_{k}$ is an irreducible $G$-module by Lemma 3. Property (1) is proven.

By Lemma 3. for every $a_{i}$ there exists $\gamma \in F$ such that for every $h \in I_{k}$ we have $a_{i} h=\gamma h+j$ where $j \in J_{k}$. Thus $c_{i} h=a_{i} h-b_{i} h=\gamma h+\left(j-b_{i} h\right)$ where $j-b_{i} h \in J_{k}$. However, $c_{i} h \in \tilde{T}_{k}$ for $h \in \tilde{T}_{k}$, and $j-b_{i} h=0$. Property (2) is proven.

By Lemma 7, each $I_{i} / J_{i}$ is a faithful $G_{i} \oplus\left\langle a_{i}\right\rangle_{F}$-module and either $a_{i}=0$ or $a_{i}$ acts on $I_{i} / J_{i}$ by a nonzero scalar operator. Thus either $a_{i}=c_{i}=0$ or $a_{i} \neq 0$ and $c_{i} \neq 0$ acts on $\tilde{T}_{i}$ by a nonzero scalar operator that belongs to the center of $\operatorname{End}_{F} \tilde{T}_{i}$. Since all homomorphic images of $G_{i}$ have zero center, each $\tilde{T}_{i}$ is a faithful $G_{i} \oplus\left\langle c_{i}\right\rangle_{F}$-module and $\operatorname{dim}\left(G_{k} \oplus\left\langle c_{k}\right\rangle_{F}\right)=\operatorname{dim}\left(G_{k} \oplus\left\langle a_{k}\right\rangle_{F}\right)$. Property (3) is proven.

Property (4) is a consequence of Property (5) of Lemma 7.

Lemma 10. Let $M$ be a finite dimensional irreducible module over a semisimple Lie algebra $G$ and $\tilde{G}$ be a Lie ideal of $G$ such that $M$ is a faithful $\tilde{G}$-module. Then there exist faithful irreducible $\tilde{G}$-submodules $\tilde{M}_{i} \subseteq M$ such that $M=\tilde{M}_{1} \oplus \tilde{M}_{2} \oplus \cdots \oplus \tilde{M}_{k}$.

Proof. There exists a semisimple ideal $\bar{G}$ such that $G=\tilde{G} \oplus \bar{G}$. By [5, Proposition 7.3.1'], $M \cong \tilde{M} \otimes \bar{M}$ for some irreducible $\tilde{G}$-module $\tilde{M}$ and irreducible $\bar{G}$-module $\bar{M}$. Let $m_{1}, \ldots, m_{k}$ be basis elements of $\bar{M}$. Then $M=\left(\tilde{M} \otimes m_{1}\right) \oplus$ $\left(\tilde{M} \otimes m_{2}\right) \oplus \cdots \oplus\left(\tilde{M} \otimes m_{k}\right)$ is the direct sum of irreducible $\tilde{G}$-modules. These submodules are faithful since $M$ is faithful. 
By Condition (2) of the definition of $d(\rho)$, there exist elements $q_{1}, \ldots, q_{r-1} \in$ $A \cup\{1\}$ such that $\tilde{T}_{1} q_{1} \tilde{T}_{2} \ldots \tilde{T}_{r-1} q_{r-1} \tilde{T}_{r} \neq 0$. Choose $n_{i} \in \mathbb{Z}_{+}$with the maximal $\sum_{i=0}^{r-1} n_{i}$ such that

$$
\left(\prod_{k=1}^{n_{0}} j_{0 k}\right) \tilde{T}_{1} q_{1}\left(\prod_{k=1}^{n_{1}} j_{1 k}\right) \tilde{T}_{2} \ldots q_{r-1}\left(\prod_{k=1}^{n_{r-1}} j_{r-1, k}\right) \tilde{T}_{r} \neq 0
$$

for some $j_{i k} \in J(A)$. Denote $\left(q_{i} \prod_{k=1}^{n_{i}} j_{i k}\right)$ again by $q_{i}$ for $1 \leqslant i \leqslant r-1$ and $\prod_{k=1}^{n_{0}} j_{0 k}$ by $q_{0}$. Then $q_{i} \in A \cup\{1\}$ and

$$
q_{0} \tilde{T}_{1} q_{1} \tilde{T}_{2} \ldots \tilde{T}_{r-1} q_{r-1} \tilde{T}_{r} \neq 0,
$$

but

$$
q_{0} \tilde{T}_{1} q_{1} \tilde{T}_{2} \ldots \tilde{T}_{k-1} q_{k-1}\left(j \tilde{T}_{k}\right) q_{k} \tilde{T}_{k+1} \ldots \tilde{T}_{r-1} q_{r-1} \tilde{T}_{r}=0
$$

for all $j \in J(A)$ and $1 \leqslant k \leqslant r$.

By Lemma 10, for every $k$ we can choose a faithful irreducible $G_{k} \oplus\left\langle c_{k}\right\rangle_{F^{-}}$ submodule $T_{k} \subseteq \tilde{T}_{k}$ such that

$$
q_{0} T_{1} q_{1} T_{2} \ldots T_{r-1} q_{r-1} T_{r} \neq 0 .
$$

Lemma 11. Let $\varphi: \bigoplus_{i=1}^{r}\left(G_{i} \oplus\left\langle a_{i}\right\rangle\right) \rightarrow \bigoplus_{i=1}^{r}\left(G_{i} \oplus\left\langle c_{i}\right\rangle\right)$ be the isomorphism defined by formulas $\varphi(g)=g$ for all $g \in G_{i}$ and $\varphi\left(a_{i}\right)=c_{i}$. Let $f_{i}$ be multilinear polynomials and $h_{1}^{(i)}, \ldots, h_{n_{i}}^{(i)} \in \bigoplus_{k=1}^{r}\left(G_{k} \oplus\left\langle a_{k}\right\rangle_{F}\right), t_{i} \in \tilde{T}_{i}$ be some elements. Then

$$
\begin{gathered}
q_{0} f_{1}\left(h_{1}^{(1)}, \ldots, h_{n_{1}}^{(1)}\right) t_{1} q_{1} f_{2}\left(h_{1}^{(2)}, \ldots, h_{n_{2}}^{(2)}\right) t_{2} \ldots q_{r-1} f_{r}\left(h_{1}^{(r)}, \ldots, h_{n_{r}}^{(r)}\right) t_{r} \\
=q_{0} f_{1}\left(\varphi\left(h_{1}^{(1)}\right), \ldots, \varphi\left(h_{n_{1}}^{(1)}\right)\right) t_{1} q_{1} f_{2}\left(\varphi\left(h_{1}^{(2)}\right), \ldots, \varphi\left(h_{n_{2}}^{(2)}\right)\right) t_{2} \\
\ldots q_{r-1} f_{r}\left(\varphi\left(h_{1}^{(r)}\right), \ldots, \varphi\left(h_{n_{r}}^{(r)}\right)\right) t_{r}
\end{gathered}
$$

In other words, we can replace $a_{i}$ with $c_{i}$ and the result does not change.

Proof. We rewrite $a_{i}=b_{i}+c_{i}=b_{i}+\varphi\left(a_{i}\right)$ and use the multilinearity of $f_{i}$. By (2), terms with $b_{i}$ vanish.

Let $m k \leqslant n$ where $m, k, n \in \mathbb{N}$ are some numbers. Denote by $Q_{m, k, n} \subseteq P_{n}$ the subspace spanned by all polynomials that are alternating in $k$ disjoint subsets of variables $\left\{x_{1}^{i}, \ldots, x_{m}^{i}\right\} \subseteq\left\{x_{1}, x_{2}, \ldots, x_{n}\right\}, 1 \leqslant i \leqslant k$.

We need the following theorem [3, Remark 12.1].

Theorem 3 (Yu.P. Razmyslov). Let $B$ be a reductive Lie algebra over an algebraically closed field of characteristic zero, and let $\operatorname{dim} B=m$. Let $\tau: B \hookrightarrow U$ be an embedding of $B$ to a simple associative algebra $U$ generated by $\tau(B)$, with a nonzero center. Then there exist $k$ and a multilinear polynomial $f \in Q_{m, k, m k} \backslash \operatorname{Id}(\tau)$ such that $f\left(\tau\left(p_{1}\right), \ldots, \tau\left(p_{m k}\right)\right)$ belongs to the center of $U$ for all $p_{i} \in B$.

Lemma 12. If $d \neq 0$, then there exist numbers $k, n_{0} \in \mathbb{N}$ such that for every $n \geqslant n_{0}$ there exist disjoint subsets $X_{1}, \ldots, X_{k \ell} \subseteq\left\{x_{1}, \ldots, x_{n}\right\}, \ell=\left[\frac{n-n_{0}}{k d}\right]$, $\left|X_{1}\right|=\ldots=\left|X_{k \ell}\right|=d$ and a polynomial $f \in P_{n} \backslash \operatorname{Id}(\rho)$ alternating on the variables of each set $X_{j}$. 
Proof. Recall that each $T_{i}$ is a faithful irreducible $G_{i} \oplus\left\langle c_{i}\right\rangle_{F}$-module. Denote by $\tau_{i}$ the embedding $G_{i} \oplus\left\langle c_{i}\right\rangle \hookrightarrow \operatorname{End}_{F}\left(T_{i}\right)$. By virtue of the density theorem [8, Section 4.3], the space $\tau_{i}\left(G_{i} \oplus\left\langle c_{i}\right\rangle\right)$ generates $\operatorname{End}_{F}\left(T_{i}\right)$ as an associative algebra. The center of $\operatorname{End}_{F}\left(T_{i}\right)$ is a linear span of its unit $\mathrm{id}_{T_{i}}$. By virtue of Theorem 3 , for some $k_{i} \in \mathbb{N}$ there exists a multilinear polynomial $f_{i} \in Q_{d_{i}, k_{i}, k_{i} d_{i}} \backslash \operatorname{Id}\left(\rho_{i}\right), d_{i}=$ $\operatorname{dim}\left(G_{i} \oplus\left\langle c_{i}\right\rangle\right)$, satisfying the following property. If we substitute any elements from $\tau_{i}\left(G_{i} \oplus\left\langle c_{i}\right\rangle\right)$ for the variables of $f_{i}$, then the result belongs to the center of $\operatorname{End}_{F}\left(T_{i}\right)$. Therefore, $\tau_{i}\left(f_{i}\left(h_{1}^{(i)}, \ldots, h_{d_{i} k_{i}}^{(i)}\right)\right)=\mathrm{id}_{T_{i}}$ for some $h_{\beta}^{(i)} \in G_{i} \oplus\left\langle c_{i}\right\rangle_{F}$ and $f_{i}\left(h_{1}^{(i)}, \ldots, h_{d_{i} k_{i}}^{(i)}\right) t_{i}=t_{i}$ for all $t_{i} \in T_{i}$. We can consider products of several copies of $f_{i}$ in different variables and assume that $k_{1}=k_{2}=\ldots=k_{r}=k$.

Equation (3) implies that

$$
b:=q_{0} t_{1} q_{1} t_{2} \ldots q_{r-1} t_{r} \neq 0
$$

for some $t_{i} \in T_{i}$. Fix these $t_{i}$. Note that $q_{i}$ are sums of $q_{i 1} \ldots q_{i \theta_{i}}$ and $t_{i}$ are sums of $t_{i 1} \ldots t_{i \xi_{i}}$ where $\theta_{i} \geqslant 0, \xi_{i}>0$ and $q_{i k}, t_{i k} \in \rho(L)$. Denote the maximal $\theta_{i}$ by $\eta_{i}$ and the maximal $\xi_{i}$ by $\zeta_{i}$. Let $n_{0}=\sum_{i=1}^{r}\left(\eta_{i-1}+\zeta_{i}\right), \ell=\left[\frac{n-n_{0}}{k d}\right]$, and $\alpha=\left[\frac{n-\ell k d}{k d_{1}}\right]+1$.

Consider the polynomial $\tilde{f}^{(\alpha)}=w_{0} f_{1}^{\alpha} \tilde{f}$ where

$$
\tilde{f}=\left(f_{1}^{\ell} z_{1}\right) w_{1}\left(f_{2}^{\ell} z_{2}\right) w_{2}\left(f_{3}^{\ell} z_{3}\right) \ldots\left(f_{r-1}^{\ell} z_{r-1}\right) w_{r-1}\left(f_{r}^{\ell} z_{r}\right) .
$$

Here the variables of all copies of $f_{i}$ are different and $f^{(\alpha)}$ is a multilinear polynomial of degree $\ell k d+2 r+\alpha k d_{1}$.

Let $w_{i}=q_{i}, z_{i}=t_{i}$. Equation (4) and the choice of $f_{i}$ imply that if we replace the variables of $f_{i}$ with $h_{\beta}^{(i)}$, then the value of $\tilde{f}^{(\alpha)}$ equals $b$. Denote this substitution of elements by $\Delta$.

Each copy of $f_{i}, 1 \leqslant i \leqslant r$, is alternating on disjoint sets $X_{1}^{i, j}, \ldots, X_{k}^{i, j}$ where $\left|X_{1}^{i, j}\right|=\ldots=\left|X_{k}^{i, j}\right|=d_{i}$ and $1 \leqslant j \leqslant \ell$ is the number of the copy of $f_{i}$ in $\tilde{f}$. Denote $X_{(j-1) k+v}=\coprod_{i=1}^{r} X_{v}^{i, j}$ for $1 \leqslant j \leqslant \ell, 1 \leqslant v \leqslant k$. Then $\left|X_{j}\right|=d$. Consider the polynomial $f^{(\alpha)}=w_{0} f_{1}^{\alpha} \operatorname{Alt}_{1} \ldots$ Alt $_{k \ell} \tilde{f}$ where the operator Alt $_{j}$ alternates a polynomial on the variables of $X_{j}$. We claim that $f^{(\alpha)}$ does not vanish under the substitution $\Delta$.

Note that the alternation does not change $z_{i}$. Thus Property (4) of Lemma 9 implies that all the terms of alternation in which variables from $X_{v}^{i, j}$ are replaced with variables from $X_{v}^{i^{\prime}, j}, i<i^{\prime}$, vanish. Hence we may consider only those terms where $X_{v}^{i, j}$ are invariant. Recall that each $f_{i}$ is alternating on $X_{v}^{i, j}$. Thus the result of the substitution $\Delta$ for the variables of $f^{(\alpha)}$ equals $\left(d_{1} ! d_{2} ! \ldots d_{r} !\right)^{k \ell} b \neq 0$. Moreover, by Lemma 11, it is not important whether we replace the variables of $f_{i}$ with $a_{i}$ or $c_{i}$. Now we assume that all the variables of $f_{i}$ are replaced with elements from $G_{i} \oplus\left\langle a_{i}\right\rangle$.

Now we rewrite $q_{i}$ as sums of $q_{i 1} \ldots q_{i \theta_{i}}$ and $t_{i}$ as sums of $t_{i 1} \ldots t_{i \xi_{i}}$ where $0 \leqslant$ $\theta_{i} \leqslant \eta_{i}, 0<\xi_{i} \leqslant \zeta_{i}$ and $q_{i k}, t_{i k} \in \rho(L)$. Using the linearity of $f^{(\alpha)}$ in $w_{i}$ and $t_{i}$, we obtain that $f^{(\alpha)}$ does not vanish under some substitution $w_{i}=q_{i 1} \ldots q_{i \theta_{i}}$ and $z_{i}=t_{i 1} \ldots t_{i \xi_{i}}$. Now we replace $w_{i}$ in $\tilde{f}$ with $y_{i 1} \ldots y_{i \theta_{i}}$ (or remove $w_{i}$ if $\theta_{i}=0$ ) and $z_{i}$ with $v_{i 1} \ldots v_{i \xi_{i}}$. Denote the polynomial obtained by $\hat{f}$. Then

$$
\hat{f}^{(\alpha)}=y_{01} \ldots y_{0 \theta_{0}} f_{1}^{\alpha} \operatorname{Alt}_{1} \ldots \operatorname{Alt}_{k \ell} \hat{f}
$$


is a multilinear polynomial of degree

$$
\ell k d+\alpha k d_{1}+\sum_{i=1}^{r}\left(\theta_{i-1}+\xi_{i}\right)
$$

and $\hat{f}^{(\alpha)} \notin \operatorname{Id}(\rho)$. Note that $\ell k d \leqslant \operatorname{deg} \hat{f} \leqslant n_{0}+\ell k d \leqslant n$ and $\operatorname{deg} \hat{f}^{(\alpha)}>\ell k d+$ $\alpha k d_{1}>n$.

Expand the first $\alpha$ copies of $f_{1}$ in $\hat{f}^{(\alpha)}$. At least one of the polynomials obtained is not a polynomial identity. Denote it by $g^{(\alpha)}$. Remove $\left(\operatorname{deg} \hat{f}^{(\alpha)}-n\right)$ initial variables in $g^{(\alpha)}$ and denote the polynomial obtained by $f$. Then

$$
f=u_{1} u_{2} \ldots u_{(n-\operatorname{deg} \hat{f})} \operatorname{Alt}_{1} \ldots \operatorname{Alt}_{k \ell} \hat{f} \in P_{n} \backslash \operatorname{Id}(\rho)
$$

satisfies all the conditions of Lemma 12, Here $u_{i}$ are some variables from $\left\{x_{1}, x_{2}, \ldots\right.$, $\left.x_{n}\right\}$.

Lemma 13. Let $k, n_{0}, \ell$ be the numbers from Lemma 12. Then for every $n \geqslant n_{0}$ there exists a partition $\lambda=\left(\lambda_{1}, \ldots, \lambda_{s}\right) \vdash n, \lambda_{i} \geqslant k \ell-C$ for every $1 \leqslant i \leqslant d$, with $m(\rho, \lambda) \neq 0$. Here $C=p((\operatorname{dim} A) p+3)((\operatorname{dim} \rho(L))-d)$.

Proof. Consider the polynomial $f$ from Lemma 12. It is sufficient to prove that $e_{T_{\lambda}}^{*} f \notin \operatorname{Id}(\rho)$ for some tableau $T_{\lambda}$ of a desired shape $\lambda$. It is known that $F S_{n}=$ $\bigoplus_{\lambda, T_{\lambda}} F S_{n} e_{T_{\lambda}}^{*}$, where the summation runs over the set of all standard tableax $T_{\lambda}$, $\lambda \vdash n$. Thus $F S_{n} f=\sum_{\lambda, T_{\lambda}} F S_{n} e_{T_{\lambda}}^{*} f \nsubseteq \operatorname{Id}(\rho)$ and $e_{T_{\lambda}}^{*} f \notin \operatorname{Id}(\rho)$ for some $\lambda \vdash n$. We claim that $\lambda$ is of a desired shape. It is sufficient to prove that $\lambda_{d} \geqslant k \ell-C$, since $\lambda_{i} \geqslant \lambda_{d}$ for every $1 \leqslant i \leqslant d$. Each row of $T_{\lambda}$ includes numbers of no more than one variable from each $X_{i}$, since $e_{T_{\lambda}}^{*}=b_{T_{\lambda}} a_{T_{\lambda}}$ and $a_{T_{\lambda}}$ is symmetrizing the variables of each row. Thus $\sum_{i=1}^{d-1} \lambda_{i} \leqslant \ell k(d-1)+(n-\ell k d)=n-\ell k$. By virtue of Lemma 6. $\sum_{i=1}^{d} \lambda_{i} \geqslant n-C$. Therefore $\lambda_{d} \geqslant \ell k-C$.

Proof of Theorem 1. The Young diagram $D_{\lambda}$ from Lemma 13 contains the rectangular subdiagram $D_{\mu}, \mu=(\underbrace{k \ell-C, \ldots, k \ell-C}_{d})$. The branching rule for $S_{n}$ implies that if we consider a restriction of $S_{n}$-action on $M(\lambda)$ to $S_{n-1}$, then $M(\lambda)$ becomes the direct sum of all nonisomorphic $F S_{n-1}$-modules $M(\nu), \nu \vdash(n-1)$, where each $D_{\nu}$ is obtained from $D_{\lambda}$ by deleting one box. In particular, $\operatorname{dim} M(\nu) \leqslant \operatorname{dim} M(\lambda)$. Applying the rule $(n-d(k \ell-C))$ times, we obtain $\operatorname{dim} M(\mu) \leqslant \operatorname{dim} M(\lambda)$. By the hook formula, $\operatorname{dim} M(\mu)=\frac{(d(k \ell-C)) !}{\prod_{i, j} h_{i j}}$, where $h_{i j}$ is the length of the hook with edge in $(i, j)$. By the Stirling formula,

$\operatorname{dim} M(\mu) \geqslant \frac{(d(k \ell-C)) !}{((k \ell-C+d) !)^{d}} \sim \frac{\sqrt{2 \pi d(k \ell-C)}\left(\frac{d(k \ell-C)}{e}\right)^{d(k \ell-C)}}{\left(\sqrt{2 \pi(k \ell-C+d)}\left(\frac{k \ell-C+d}{e}\right)^{k \ell-C+d}\right)^{d}} \sim C_{5} \ell^{r_{5}} d^{d k \ell}$

as $\ell \rightarrow \infty$ for some constants $C_{5}>0, r_{5} \in \mathbb{Q}$. Since $\ell=\left[\frac{n-n_{0}}{k d}\right]$, this gives the lower bound. The upper bound has been proved in Theorem 2 , 


\section{EXAMPLES}

Example 2. Let $\rho: L \rightarrow \mathfrak{g l}(V)$ be a finite dimensional representation of a Lie algebra $L$ over a field $F$. If $\rho(a)$ is a nilpotent operator for any $a \in L$, then $c_{n}(\rho)=0$ for $n \geqslant \operatorname{dim} V$. Conversely, if $c_{n}(\rho)=0$ for some $n \in \mathbb{N}$, then $\rho(L)$ generates a nilpotent associative subalgebra $A$ in $\operatorname{End}_{F}(V)$.

Proof. Suppose $L$ acts on $V$ by nilpotent operators. One of the variants of the Engel theorem [9, Corollary from Theorem 3.3] implies that there exists a basis in $V$ in which all the operators $\rho(a), a \in L$, have strictly upper triangular matrices. The associative algebra of strictly upper triangular matrices is nilpotent and $\rho\left(a_{1}\right) \rho\left(a_{2}\right) \ldots \rho\left(a_{n}\right)=0$ in $\operatorname{End}_{F}(V)$ for all $a_{i} \in L$ and $n \geqslant \operatorname{dim} V$. Thus $c_{n}(\rho)=0$.

Suppose $c_{n}(\rho)=0$ for some $n \in \mathbb{N}$. Then $\rho\left(a_{1}\right) \rho\left(a_{2}\right) \ldots \rho\left(a_{n}\right)=0$ for all $a_{i} \in L$. Hence $A^{n}=0$.

Example 3. Let $\rho: L \rightarrow \mathfrak{g l}(V)$ be a finite dimensional irreducible representation of a Lie algebra $L$ over an algebraically closed field $F$ of characteristic 0 . Then there exist constants $C>0, r \in \mathbb{R}$ such that

$$
C n^{r}(\operatorname{dim} \rho(L))^{n} \leqslant c_{n}(\rho) \leqslant(\operatorname{dim} V)^{2}(\operatorname{dim} \rho(L))^{n} \text { for all } n \in \mathbb{N} .
$$

Proof. Consider polynomials as $n$-linear maps from $\rho(L)$ to $\operatorname{End}_{F} V$. Then we have the natural map $P_{n} \rightarrow \operatorname{Hom}_{F}\left((\rho(L))^{\otimes n} ; \operatorname{End}_{F} V\right)$ with the kernel $P_{n} \cap \operatorname{Id}(\rho)$ that leads to the embedding

$$
\frac{P_{n}}{P_{n} \cap \operatorname{Id}(\rho)} \hookrightarrow \operatorname{Hom}_{F}\left((\rho(L))^{\otimes n} ; \operatorname{End}_{F} V\right) .
$$

Thus

$c_{n}(\rho)=\operatorname{dim}\left(\frac{P_{n}}{P_{n} \cap \operatorname{Id}(\rho)}\right) \leqslant \operatorname{dim} \operatorname{Hom}_{F}\left((\rho(L))^{\otimes n} ; \operatorname{End}_{F} V\right)=(\operatorname{dim} V)^{2}(\operatorname{dim} \rho(L))^{n}$

and we obtain the upper bound. Hence $d(\rho) \leqslant \operatorname{dim} \rho(L)$. Since $V$ is an irreducible $L$-module, $V$ is an irreducible $A$-module. The density theorem [8, Section 4.3] implies

$$
A=\operatorname{End}_{F}(V) \cong M_{k}(F), \quad k=\operatorname{dim} V .
$$

Let $I=\left\langle e_{11}, e_{21}, \ldots, e_{k 1}\right\rangle \subseteq A$ and $J=0$. Then $I$ is a minimal left ideal of $A$ and $I / J$ is a faithful irreducible $A$-module. Thus $\operatorname{Ann}(I / J)=0$ and

$$
\operatorname{dim}(\rho(L) /(\rho(L) \cap \operatorname{Ann}(I / J)))=\operatorname{dim}(\rho(L)) .
$$

Hence $d(\rho) \geqslant \operatorname{dim}(\rho(L))$. This yields the lower bound.

Example 4. Let $\rho: L \rightarrow \mathfrak{g l}(V)$ be a finite dimensional completely reducible representation of a Lie algebra $L$ over an algebraically closed field $F$ of characteristic 0 , i.e.

$$
V=\bigoplus_{i=1}^{s} \bigoplus_{j=1}^{k_{i}} V_{i j},
$$

where each $V_{i j}$ is irreducible and $V_{i j} \cong V_{k l}$ if and only if $i=k$. Denote $\rho_{i j}: L \rightarrow$ $\mathfrak{g l}\left(V_{i j}\right), \rho_{i j}(a)=\left.\rho(a)\right|_{V_{i j}}, 1 \leqslant i \leqslant s, 1 \leqslant j \leqslant k_{i}, a \in L$. Denote

$$
d=\max _{\substack{1 \leqslant i \leqslant s, 1 \leqslant j \leqslant k_{i}}}\left(\operatorname{dim} \rho_{i j}(L)\right) .
$$


Then there exist constants $C_{1}, C_{2}>0, r_{1}, r_{2} \in \mathbb{R}$ such that

$$
C_{1} n^{r_{1}} d^{n} \leqslant c_{n}(\rho) \leqslant C_{2} n^{r_{2}} d^{n} \text { for all } n \in \mathbb{N} .
$$

Proof. Let $A$ be the associative subalgebra of $\operatorname{End}_{F}(V)$ generated by $\rho(L)$. Note that

$$
\Delta=\operatorname{End}_{A}(V) \cong M_{k_{1}}(F) \oplus \ldots \oplus M_{k_{s}}(F) .
$$

Indeed, denote by $\varphi_{i j}$ the isomorphism $V_{i j} \rightarrow V_{i 1}$ and by $p_{i j}$ the projection $V \rightarrow V_{i j}$. Let $\psi \in \Delta$. Then by Schur's lemma $\varphi_{k \ell} p_{k \ell} \psi \varphi_{i j}^{-1}: V_{i 1} \rightarrow V_{k 1}$ is a scalar operator for $i=k$ and is a zero operator for $i \neq k$. Define $\alpha_{\ell j}^{(i)}(\psi) \in F$ by the formula $\varphi_{i \ell} p_{i \ell} \psi \varphi_{i j}^{-1}(a)=\alpha_{\ell j}^{(i)}(\psi) a$ for all $a \in V_{i 1}$. Then $\Phi: \Delta \rightarrow M_{k_{1}}(F) \oplus \ldots \oplus M_{k_{s}}(F)$, $\Phi(\psi)=\left(\left(\alpha_{\ell j}^{(1)}(\psi)\right),\left(\alpha_{\ell j}^{(2)}(\psi)\right), \ldots,\left(\alpha_{\ell j}^{(s)}(\psi)\right)\right)$ is an isomorphism and

$$
\Phi^{-1}\left(\left(\beta_{\ell j}^{(1)}\right),\left(\beta_{\ell j}^{(2)}\right), \ldots,\left(\beta_{\ell j}^{(s)}\right)\right)=\sum_{i=1}^{s} \sum_{j, \ell=1}^{k_{i}} \beta_{\ell j}^{(i)} \varphi_{i \ell}^{-1} \varphi_{i j} p_{i j} .
$$

Here $\left(\beta_{\ell j}^{(i)}\right) \in M_{k_{i}}(F)$ are arbitrary matrices.

By the density theorem [8, Section 4.3], $A=\operatorname{End}_{\Delta}(V)$. Thus

$$
A=\operatorname{End}_{\Delta}(V) \cong \operatorname{End}_{F}\left(V_{11}\right) \oplus \operatorname{End}_{F}\left(V_{21}\right) \oplus \ldots \oplus \operatorname{End}_{F}\left(V_{s 1}\right),
$$

where $\left(\psi_{1}, \ldots, \psi_{s}\right)$ acts on $V_{i j}$ by the operator $\varphi_{i j}^{-1} \psi_{i} \varphi_{i j}$. Indeed, every operator $\psi \in \operatorname{End}_{\Delta}(V)$ must commute with $p_{i j} \in \Delta$. Hence $V_{i j}$ are invariant under $\psi$. Moreover, $\psi$ must commute with $\varphi_{i j}^{-1} \varphi_{i \ell} p_{i \ell}$. Therefore,

$$
\begin{aligned}
\psi\left(\varphi_{i j}^{-1} \varphi_{i \ell} p_{i \ell}\right) & =\left(\varphi_{i j}^{-1} \varphi_{i \ell} p_{i \ell}\right) \psi \\
\varphi_{i j}\left(\psi \varphi_{i j}^{-1} \varphi_{i \ell} p_{i \ell}\right) \varphi_{i \ell}^{-1} & =\varphi_{i j}\left(\varphi_{i j}^{-1} \varphi_{i \ell} p_{i \ell} \psi\right) \varphi_{i \ell}^{-1}
\end{aligned}
$$

and $\varphi_{i j} \psi \varphi_{i j}^{-1}=\varphi_{i \ell} \psi \varphi_{i \ell}^{-1}$ for all $1 \leqslant i \leqslant s, 1 \leqslant j, \ell \leqslant k_{i}$. We obtain the isomorphism

$$
\begin{gathered}
\Xi: A \rightarrow \operatorname{End}_{F}\left(V_{11}\right) \oplus \operatorname{End}_{F}\left(V_{21}\right) \oplus \ldots \oplus \operatorname{End}_{F}\left(V_{s 1}\right), \\
\Xi(\psi)=\left(\left.\psi\right|_{V_{11}},\left.\psi\right|_{V_{21}}, \ldots,\left.\psi\right|_{V_{s 1}}\right) .
\end{gathered}
$$

If $I$ is a left ideal in $A$, then $I=I_{1} \oplus \ldots \oplus I_{s}$, where $\Xi\left(I_{i}\right)$ is a left ideal in $\operatorname{End}_{F}\left(V_{i 1}\right)$. Consider arbitrary left ideals $I_{1}, \ldots, I_{r}, J_{1}, \ldots, J_{r}$ of $A$ that satisfy Conditions (1)-(2). There exist numbers $1 \leqslant i_{1}, \ldots, i_{r} \leqslant s$ such that $I_{k}=\tilde{I}_{k} \oplus \hat{I}_{k}$, $J_{k}=\tilde{J}_{k} \oplus \hat{I}_{k}$, where $\tilde{I}_{k}$ and $\tilde{J}_{k}$ are left ideals of $\Xi^{-1}\left(\operatorname{End}_{F}\left(V_{i_{k} 1}\right)\right)$ and $\hat{I}_{k}$ is a left ideal of $\Xi^{-1}\left(\bigoplus_{\ell \neq i_{k}} \operatorname{End}_{F}\left(V_{\ell 1}\right)\right)$. Hence $\operatorname{Ann}\left(I_{k} / J_{k}\right)=\Xi^{-1}\left(\bigoplus_{\ell \neq i_{k}} \operatorname{End}_{F}\left(V_{\ell 1}\right)\right)$. Thus $\operatorname{Ann}\left(I_{k} / J_{k}\right) \cap \rho(L)=\operatorname{ker} \rho_{i_{k} 1}$ and $\operatorname{dim}\left(\rho(L) /\left(\operatorname{Ann}\left(I_{k} / J_{k}\right) \cap \rho(L)\right)\right)=\operatorname{dim} \rho_{i_{k} 1}(L)$. Choose $A$-submodules $T_{k}$ such that $\tilde{I}_{k}=T_{k} \oplus \tilde{J}_{k}$. If $i_{k} \neq i_{\ell}$ for some $k$ and $\ell$, then $T_{1} q_{1} T_{2} \ldots T_{r-1} q_{r-1} T_{r}=0$ for all $q_{1}, \ldots, q_{r-1} \in A \cup\{1\}$. Thus $i_{1}=\ldots=i_{r}$ and $d(\rho)=\max _{\substack{1 \leqslant i \leqslant s, 1 \leqslant j \leqslant k_{i}}} \operatorname{dim} \rho_{i j}(L)$. By Theorem 1 we obtain the bounds. 
Example 5. Let $\rho: L \rightarrow \mathfrak{g l}(V)$ be a nontrivial finite dimensional representation of a simple Lie algebra $L$ over an algebraically closed field $F$ of characteristic 0 . Then there exist constants $C_{1}, C_{2}>0, r_{1}, r_{2} \in \mathbb{R}$ such that

$$
C_{1} n^{r_{1}}(\operatorname{dim} L)^{n} \leqslant c_{n}(\rho) \leqslant C_{2} n^{r_{2}}(\operatorname{dim} L)^{n} \text { for all } n \in \mathbb{N} \text {. }
$$

Proof. By the Weyl theorem, $\rho$ is completely reducible. Let $\rho_{i}: L \rightarrow \mathfrak{g l}\left(V_{i}\right)$ be the corresponding irreducible representations. Then Example 4 implies that

$$
d(\rho)=\max _{1 \leqslant i \leqslant s} \operatorname{dim} \rho_{i}(L)=\operatorname{dim} L .
$$

\section{ACKNOWLEDGEMENTS}

The author is grateful to Yuri Bahturin and Mikhail Kotchetov for helpful discussions.

\section{REFERENCES}

[1] Giambruno, A., Zaicev, M. V. (2005) Polynomial identities and asymptotic methods. AMS Mathematical Surveys and Monographs Vol. 122, Providence, RI, 352 pp. MR2176105 (2006g:16054)

[2] Zaitsev, M. V. (2002) Integrality of exponents of growth of identities of finite-dimensional Lie algebras. Izv. Math. 66, 463-487. MR.1921808 (2003g:17004)

[3] Razmyslov, Yu. P. (1994) Identities of algebras and their representations, Transl. Math. Monogr., vol. 138, Amer. Math. Soc., Providence, RI. MR 1291603 (95i:16022)

[4] Plotkin, B. I., Vovsi, S. M. (1983) Varieties of group representations: general theory, connections, and applications. "Zinante", Riga (Russian). MR739330 (86e:20001)

[5] Goto, M., Grosshans, F. (1978) Semisimple Lie algebras. Mercel Dekker, New York and Basel. MR0573070 (58:28084)

[6] Bakhturin, Yu. A. (1987) Identical relations in Lie algebras. VNU Science Press, Utrecht. MR886063(88f:17032)

[7] Drensky, V.S. (2000) Free algebras and PI-algebras: graduate course in algebra. SpringerVerlag, Singapore. MR.1712064 (2000j:16002)

[8] Jacobson, N. (1989) Basic algebra II. Second edition. W.H. Freeman and Company, New York. MR:1009787 (90m:00007)

[9] Humphreys, J.E. (1978) Introduction to Lie algebras and representation theory. SpringerVerlag, New York. MR499562 (81b:17007)

Memorial University of Newfoundland, St. John's, NL, Canada

E-mail address: alexey.gordienko@vub.ac.be 